\title{
Animación hotelera, una perspectiva de diferenciación de marcas. Bases conceptuales y metodológicas.
}

\section{Hotel entertainment, a perspective of brand differentiation. Conceptual and methodological bases.}

Judith Sánchez Romero. ${ }^{1}$

\begin{abstract}
.
The present work comprises the results of the theatrical analysis implemented on the fundamental criteria that have been released up to this moment about the phenomenon of touristic animation and animation as a product in establishments of touristic lodging that are visible on the market under a brand, which determined in reflexing about the redesigned of one of the brands that operates the Cuban hotelier group Cubanacán, Club Amigo, taking into consideration one of the problematics that are shown in the hotelier activities of the country, the no differentiation on the market of a great deal of the designed brands, even though when the corresponding concept had been previously established, that's why when establishing the methodological and conceptual basis and the antecedents of the research is the general objective, using theoretical methods such as :the analysis and the syntax, induction and deduction, logical-historical, bibliographic and documental revision, that allowed to clarify the ways to give solution to the problem. Virtual education is a viable new form of education that comes to meet the needs, precariousness of face-to-face education and the misuse of educational technology.
\end{abstract}

Keywords: touristic and hotelier animation, product of animation, redesign of the conceptualization of the brand.

\section{Resumen.}

El presente trabajo abarca los resultados del análisis teórico realizado sobre los criterios fundamentales emitidos referente al fenómeno de la animación turística y el producto animación en establecimientos de alojamiento turístico que se visualizan frente al mercado bajo una marca, lo que determinó en reflexionar sobre el rediseño de una de las marcas que opera el grupo hotelero Cubanacán, Club Amigo, tomando en consideración una de las problemáticas que se evidencian

\footnotetext{
${ }^{1}$ Universidad de la Habana. Cuba, yudith.sanchez@ftur.uh.cu
} 
en la actividad hotelera en Cuba sobre, la no diferenciación en el mercado de una gran parte de las marcas diseñadas, aun cuando el Concepto de estas, se haya establecido con anterioridad, por lo que establecer las bases conceptuales, metodológicas y los antecedentes de la investigación, es el objetivo general de la misma, empleándose para ello métodos teóricos como: el análisis y síntesis, inducción/deducción, histórico-lógico, la revisión bibliográfica y documental, que permitieron sustentar dicho estudio.

Palabras claves: Animación turística y hotelera, producto animación, rediseño de conceptualización de marca.

\section{Introducción}

Partir de la premisa que el turismo, se ha convertido en una de las principales fuentes de ingresos de la economía cubana, constituye uno de los pilares fundamentales en la espiral creciente del mismo, representando una fortaleza para la actividad de animación.

Varios diagnósticos realizados en las principales cadenas hoteleras cubanas, desde el año 2012 hasta la fecha; año donde se da inicio al estudio de efectividad de la animación; concluye que de un 73,3\% de satisfacción obtenido en ese año, se avanzó paulatinamente hasta cerrar el año 2016 con un $81.7 \%$. (Pastor, 2017)

Todo lo anterior, demuestra un ascenso moderado, sin embargo, en la actualidad, la animación se encuentra en una meseta, que tiende a declinar. La pertinencia de este trabajo, descansa en el estudio exhaustivo de la actividad de animación como una de las formas de contribuir al incremento, tanto de los ingresos del sector hotelero, como el nivel de satisfacción de los visitantes al país. Atendiendo, al papel preponderante que juega la animación en los hoteles, con un concepto actualizado del entretenimiento, lo que está constituyendo, en estos momentos, uno de los motivos para elegir el producto hotelero.

El hecho de que los establecimientos de alojamiento turístico, responden, generalmente, a una marca que sale al mercado, resulta obligatorio, en dicho estudio, tomar en consideración el fenómeno marcario, en tanto, la marca condiciona, en gran medida, la manera de operar el producto.

Otro aspecto a destacar es la no correspondencia directa entre la marca y la actividad de animación en los establecimientos hoteleros, los programas de animación de la mayoría de estas entidades, ya sean de sol y playa, ciudad y/o naturaleza, están diseñados con contenidos muy parecidos, es decir, generalmente las actividades pueden ser las mismas, desarrolladas en cualquier espacio turístico, por lo que no reflejan los atributos que la marca sugiere, para aquellos que no la conocen, ni despiertan el interés para los que la conocen. Lo cual constituye el problema a investigar.

Este estudio abarca los referentes teóricos que sustentan la actividad de animación tomando en consideración el rediseño de marcas a partir del análisis de la marca Club Amigo del grupo hotelero 
Cubanacán, por ello el objetivo general de este trabajo se define en: Establecer las bases conceptuales, metodológicas y los antecedentes de la investigación que sustente el rediseño de la conceptualización de la marca Club Amigo del Grupo Cubanacán, desde la perspectiva de la animación.

Lo novedoso del trabajo radica en que, hasta el momento, a pesar que se han realizado varios estudios e investigaciones sobre la animación en Cuba, estos no han estado encaminados a emitir criterios y juicios orientados al rediseño del Concepto determinado por los beneficios y los atributos que sugieren las marcas en el mercado, desde la perspectiva de la animación, para diferenciar las mismas.

Se obtuvo que los docentes perciben como herramientas principales y de mayor uso a la distribución de material, encargar actividades individuales, mandar y recibir mensajes, encargar actividades en equipo y retroalimentar y responder dudas; el nivel de uso de las herramientas tecnológicas es alto con un porcentaje de $38.3 \%$, el nivel medio es de $31.3 \%$ y bajo es de $30.5 \%$.

\section{Metodologia}

\section{El producto animación en establecimientos de alojamiento turístico. Bases conceptuales y metodológicas}

En aras de sustentar el trabajo investigativo y de establecer el estado del arte en el cual nos encontramos, se establecen los antecedentes que existen sobre el objeto de estudio.

Para la consecución del objetivo del presente trabajo, se utilizó el método teórico, empleándose los procedimientos de la inducción-deducción, análisis-síntesis, los cuales permitieron profundizar en el conocimiento de las principales características y regularidades de la actividad, sobre la base de juicio, enfoques y criterio.

El método histórico-lógico, se utilizó para describir la evolución del fenómeno de la animación hasta los momentos actuales, pero sin llevar un orden cronológico, sino que se analizan los diferentes enfoques para llegar al fin que se quiere alcanzar. Se emplearon, además la exhaustiva revisión bibliográfica y documental, lo que permitió la búsqueda de la información pertinente y lograr las comparaciones que fueron analizadas.

A través del análisis de las definiciones emitidas por diversos autores, la autora de este trabajo pudo establecer un pensamiento más claro sobre dos términos que se usan indistintamente: la animación turística y la animación hotelera, aunque se desarrollan en distintos contextos, no quiere decir que no signifiquen lo mismo, además de analizar el fenómeno desde la perspectiva del rediseño, lo cual quedó reflejado en el presente informe de investigación. 


\section{La actividad de animación. Definiciones}

Cualquier definición de animación, debe partir, en nuestro criterio, de la ofrecida por el Diccionario de la Lengua Española (2009), el cual le otorga un doble origen etimológico: por una parte, procede de ánima, que significa vida, sentido o aliento; $\mathrm{y}$, por otra, de animus, que significa motivación, movimiento o dinamismo.

Su origen se debe buscar en el objeto de su intervención: lo cultural como eje sobre lo que progresa la humanidad, sin embargo, el desarrollo de esta acción, trajo consigo, una evolución de sus definiciones, incorporándole determinados atributos como actividad del hombre, por lo que se parte del criterio del autor que la defina y el entorno temporal en que se ubica.

Puertas (2010) define la animación como una "... serie de acciones realizadas por uno o varios individuos sobre un grupo de personas con dos objetivos, el primero desarrollar la comunicación, fomentando la vida social y la cultura en el seno de un establecimiento turístico, creando un ambiente idóneo y duradero y el segundo dinamizar y promover la economía de la instalación”.

Resulta interesante, como este autor, vincula en su definición, el aspecto sociocultural de la animación, con lo empresarial y económico, aportándole a esta actividad valor para la empresa.

Saavedra Chávez. A (2010) define la animación como el "arte de la persona de accionar, crear, promocionar, proporcionar, activar, mover, facilitar, motivar, proceder e involucrar a otra persona o grupos de estas a través de técnicas conductuales que propicien, desarrollen, contribuyan y faciliten pasar de un estado pasivo al activo provocando la participación alegre, divertida y saludable del implicado en la actividad que ocurre en su entorno, obteniendo como resultado la satisfacción”. Para Saavedra, la actividad de animación, descansa, esencialmente, en la interacción entre personas, buscando su satisfacción mediante el involucramiento de estos.

Los autores Puertas y Saavedra, de alguna manera, coinciden en que la animación es, básicamente acción, a través de técnicas que fomenten la comunicación y el intercambio entre los individuos en ambientes saludables que, al mismo tiempo, provoque satisfacción y experiencias memorables.

Sin embargo, el Manual Técnico-Metodológico para la Actividad de Animación, del Instituto Nacional de Turismo (1986), se define la animación como: “...Un conjunto de técnicas derivadas de la existencia de la recreación como un fenómeno orgánico de carácter biológico y social del hombre, que respaldada por una base científica se organizan y desarrollan en función de satisfacer los intereses de carácter multivariados que presentan los turistas".

Era entonces, cuando comenzaba a nacer este fenómeno en Cuba y como fruto de su desarrollo Abreu (2007:1) plantea que la animación "es un conjunto de técnicas derivadas de la recreación que permiten, planificar, organizar y desarrollar diferentes actividades o juegos, con el objetivo de crear un ambiente favorable en un grupo, contribuyendo al incremento de las relaciones 
sociales en el mismo y a la satisfacción de los intereses y necesidades de las personas en su tiempo libre".

Si bien hay un espacio de tiempo bastante grande entre un pronunciamiento y otro, las dos afirman que la animación es un conjunto de técnicas derivadas de la recreación, lo cual es del criterio de esta autora que este es un aspecto preciso a destacar. Un análisis más detallado sería partir de la definición de "técnicas" como la precisan diccionarios de la lengua española consultados (según ediciones de distintos años) como "el conjunto de procedimientos de un arte o ciencia. Pericia o habilidad para usar esos procedimientos".

Si técnica quiere decir lo anterior expuesto, esta autora opina que la animación por sí sola, puede considerarse un arte, en tanto contiene saberes, es en sí misma, la combinación de muchos conceptos que se interrelacionan dentro de la ciencia, como parte de las expresiones artísticas, pero con características propias, basada en técnicas y metodologías que la sustentan, sin negar que aunque la recreación y la animación están relacionadas entre sí, cada una puede gestionarse independientemente.

Como se observa, estas definiciones, un tanto genéricas, al ubicarse en un contexto específico, el turístico y más particularmente, el hotelero, adquieren atributos que le van aportando otra dimensión (económica, empresarial, turística...) sin perder, claro está, su esencia. En este sentido, se estaría hablando de la animación turística y/o la animación hotelera, cuyas particularidades enriquecen el Concepto de animación.

\section{Animación turística y animación hotelera}

De acuerdo con la Organización Mundial del Turismo (OMT), la animación turística surge como respuesta a la necesidad de los visitantes de un destino turístico, de ocupar su tiempo libre y de ocio para realizar alguna actividad que incremente su nivel de satisfacción. La referencia a esta actividad, implica estar tomando en consideración, el alcance de su actuación. El espacio en que se manifiesta la misma abarca todo el destino.

Se estaría hablando, entonces, de entidades como el aeropuerto, las carreteras, los viales de la ciudad, y lugares culturales, históricos y naturales que están enclavados tanto en el destino como, en la localidad en cuestión, que, aunque no son entidades turísticas, están vinculada a dicha actividad.

La animación turística, busca dar vida a todos los recursos que posee el destino, de forma que se haga más atractivo a los ojos del visitante, esto sugiere que guarda un estrecho vínculo con el entorno donde se manifiesta. 
La Escuela Nacional de Animación Turística (ENATUR) se planteó la definición como “...un sistema de pensamiento y acciones que proyectan la identidad nacional, influyendo en la planificación y organización integral del producto turístico, a fin de satisfacer las necesidades, intereses y motivaciones de los clientes, y propiciar su participación activa en la recreación física $y$ mental. ${ }^{2}$ Esta definición, a juicio de esta autora, constituye una de las más completas, en tanto, incorpora el enfoque sistémico, asociado a lo auténtico del país (identidad nacional), al involucramiento total y consciente del cliente.

Otro aspecto interesante de la definición que expone esta institución, es lo que se refiere al tema económico, al plantear que la animación “...constituye una ventaja competitiva que acciona en la generación de ingresos". 3

Si bien, este planteamiento guarda una lógica aceptable, a juicio de esta autora, hace reflexionar acerca de, lo que se viene disertando desde principio de siglo por diferentes especialistas reconocidos en la materia, sobre los aspectos que le faltan a la animación para que sea reconocida como un producto competitivo.

Si ventaja competitiva significa brindar en el mercado mayor valor que el resto de los competidores, entonces, para que la animación turística, cumpla esta competitividad, tendría que tener como condición, primero ser diferenciadora, satisfacer plenamente a los clientes (en consecuencia, se incrementaría la repitencia, por tanto, los ingresos) y/o estar vinculada a actividades que generen ingresos. La animación como actividad, genera ingresos de manera indirecta, de forma general y reduce gastos, es indiscutible que implica satisfacción.

Este análisis, además, está sugiriendo de manera muy clara, que la definición dada, al referirse a "ingresos", la ubica dentro de un establecimiento, en este caso, turístico.

Sobre esta base, la autora considera definir a la animación turística, como el arte de combinar diversas acciones de tipo recreativa, intelectual y social, proyectando la cultura de un país, destino o región, con el fin de brindar mayor valor al mercado.

No obstante, el análisis realizado, indica que, al abarcar la animación turística, prácticamente todo el destino, está incorporando, de alguna manera, la animación que se desarrolla dentro de los establecimientos de alojamiento, que forman parte del mismo.

A este punto, entonces se hace necesario, particularizar en esta animación a la cual se reconoce como "animación hotelera", sin llegar a confundir que se están separando los términos, sino que cada una, asume ciertas responsabilidades y funciones según el ámbito en que se desarrollen.

2Definición ofrecida por los profesores de técnicas de animación de la Escuela Nacional de Animación Turística en el año 2000.

${ }^{3}$ lbídem 
La OMT (1985) definió a la animación hotelera como el conjunto de actividades diurnas y nocturnas que se desarrollan en un establecimiento, designadas a satisfacer las necesidades de ocio, de disfrute y de esparcimiento de los huéspedes alojados en él.

Por tanto, la animación hotelera guarda diferencias con la animación turística, al estar más directamente vinculada al turista dentro de este límite de espacio asociado a un establecimiento hotelero.

Para la autora de este trabajo, la animación hotelera puede considerarse como un sistema de acciones articuladas de esparcimiento, en el establecimiento de alojamiento turístico, que se manifiestan a través de técnicas, a los fines de comunicar valores artísticos y patrimoniales, dinamizando la cultura y haciendo posible el vínculo del hotel al entorno, propicia el enriquecimiento espiritual de los turistas y potencialmente, puede accionar como un factor competitivo, generando directa o indirectamente, ingresos.

El desarrollo de la animación hotelera, fue consolidándose de una manera vertiginosa, sobre todo, a finales del siglo XX. Uno de sus mayores exponentes, a nivel mundial, es la cadena hotelera francesa, Club Mediterránea (Club Med), después de la Segunda Guerra Mundial, sus fundadores vieron la necesidad de crear un ambiente de relajación y entretenimiento, apostando por símbolos populares como convivencia, afición por lo festivo, organizadores amables conocidos como los GO (GentilsOrganisateurs), lo que condujo a que basara su estrategia operativa en un fuerte trabajo de animación, como elemento diferenciador en el mercado, a partir de la singularidad del Todo incluido.

Buscar la diferenciación, sobre la base de una entrega de actividades atractivas, donde se involucren los recursos del entorno y del hotel, pone al establecimiento en condiciones ventajosas para su elección de compra por el cliente.

Todo lo anterior implica pensar en esta actividad, como un producto, aún y cuando sea un área más del hotel, dicho de otra manera, es posible transformar la animación hotelera, en un producto.

\section{La animación hotelera como producto}

Visualizar la animación hotelera como un producto, obliga a tomar en consideración, lo que, genéricamente, se define como producto, a partir de un conjunto de aspectos formales y valores añadidos, según Parreño y Casado (2008). En este sentido, puede hablarse de cuatro elementos que configuran un producto, desde el punto de vista general:

1. La función básica,

2. las características tangibles,

3. los servicios conexos,

4. y los elementos intangibles. 
La función básica, constituye el servicio esencial que el consumidor espera del producto, para satisfacer la necesidad básica. Las características tangibles, son las características físicas que se perciben. Se distinguen tres grandes tipos de características: Características técnicas (incluye entre otros aspectos, las normas técnicas, la gama de productos); cuando estas características son percibidas por el consumidor, pueden servir de base para la diferenciación del producto frente a los competidores.

El otro grupo de características son las funcionales, que están referidas a la forma en que se presenta el producto. Aquí se incluyen aspectos como el acondicionamiento, envase, el etiquetado, entre otros. Se utilizan en la identificación del producto con la marca y en la diferenciación respecto a los competidores.

El tercer y último grupo son las características estéticas, que representan el aspecto externo del producto, el diseño óptimo del mismo que combine al mismo tiempo, funcionalidad y belleza, que le permita diferenciarse frente a otros productos competidores.

Los servicios conexos, son todos los servicios incorporados al producto, que lo enriquecen y que suponen un valor añadido para el consumidor, son, en definitiva, las formas de entrega del producto.

Los elementos intangibles refieren, al significado que los consumidores llegan a apreciar en el producto, que facilita su diferenciación frente a otros competidores por los beneficios que le brinda, este significado simbólico puede llegar a determinar la compra.

Sin embargo, cuando se realiza un análisis del producto-animación, se deben considerar, además, los siguientes aspectos:

1. Los espacios a utilizar.

2. Las actividades a desarrollar. El diseño de estas actividades debe tomar en consideración, elementos tales como:

- Capacidad de carga de los recursos naturales, culturales, sociales y humanos.

- Los productos-animación competidores.

- Tipos, estilos de animación y sus segmentos de clientes.

- Diferencias sobre el contenido de los productos-animación.

3. Los recursos materiales a utilizar para las actividades de animación.

4. Los servicios del hotel que pueden asociarse a estas actividades.

5. La promoción de las actividades.

6. La calidad, tanto de las actividades de animación como de los servicios asociados a las mismas.

7. El entorno natural, social y físico.

8. Estructura del equipo de animación.

9. Nivel de desarrollo económico y social de la localidad turística.

10. Políticas medioambientales.

11. Productos de animación, como productos-servicios periféricos del producto principal. 
Considerar la animación hotelera, como un producto, implica tener presente los grandes objetivos de la misma, en este contexto (hotel o establecimiento alojativo), estos se concentran en lo fundamental, en el descubrimiento y el involucramiento de cada individuo en las actividades encaminado a la satisfacción, así como, el incremento de los niveles de rentabilidad de la entidad de alojamiento. Son estos objetivos los que van a condicionar, mayor nivel de fidelización del cliente, convirtiéndolo en el mejor comercial del establecimiento.

El hecho de transformarse en un elemento decisor de compra, no justifica interpretar la animación como un complemento del producto-hotel, sino más bien, una parte consustancial del mismo. Ello explica claramente, que es totalmente erróneo considerar la animación del hotel como una actividad complementaria para el entretenimiento del cliente, sino que forma parte de sus áreas operativas.

Cabe sugerir que el posicionamiento de los productos/animación se deriva de las ideas generadas por los turistas potenciales y reales (imagen de marca) con respecto a los atributos reales del producto en cuestión, además de los beneficios que se exponen en las estrategias plasmadas por la empresa como parte del triángulo de servicio y que el cliente es capaz de percibir. Todo ello está dado por el valor que los clientes reciben de dicho producto y son parte de las necesidades que se han detectado en el mercado.

Al enfatizar el aspecto decisor que tiene el producto animación, es posible diseñar diferentes productos-animación en correspondencia con la demanda que recíbela entidad en cuestión.

Vinculado a lo anterior según De la Tejera (2008) existen modelos de producto-animación que se mueven a través de programas tales como los alternativos adaptados a las características de los distintos segmentos de clientela (parejas, individuales, familias). Este modelo se usa mucho en espacios turísticos de sol y playa.

La animación tematizada con una fuerte variedad de temas y representativa de la cultura del país donde se desarrolle, provocan la participación de los turistas en cualquier espacio que se desarrolle, es muy propio de hoteles y clubes todo incluido.

Otros como modelos destinados a los hoteles de ciudad donde se vinculan los programas culturales del entorno citadino con los turistas del hotel. Los programas de animación son diseñados en congruencia con las instituciones socioculturales del lugar.

La animación segmentada especializada está dirigida a segmentos que requieren de ciertos niveles de exigencia con referencia al tipo de animación (niños y adolescentes, familias, adulto mayor, negocios, salud, discapacitados, ecoturismo, deportes recreativos). Este modelo se desarrolla 
fundamentalmente en apartamentos y villas de playa, turismo de naturaleza, hoteles spa, turismo deportivo.

Puede pensarse también en una oferta de productos-animación opcionales asociados a turismo de eventos, negocios e incentivos que pueden ser un espectáculo tematizado; rutas de exploración y descubrimiento con animación, eventos deportivos-recreativos y de entretenimiento especiales, destinado a disimiles ofertas recreacionales.

Asumir la animación como un producto, implica realizar su gestión teniendo en cuenta tres ideas básicas:

1. Puede convertirse en un factor de comercialización

2. El impacto que esta actividad tiene en el uso de los recursos turísticos.

3. La animación no es un valor agregado del producto hotel y mucho menos, un complemento de éste.

En este sentido se concluye que, la animación hotelera constituye un elemento diferenciador de la oferta turística, ya que satisface las necesidades recreativas y culturales del cliente, mediante un sistema de acciones basado en principios éticos y valores de nuestra cultura e identidad nacional, atendiendo a segmentos de clientes, a través de diversos productos-animación que se trasforman en programas para todos los gustos y preferencias de estos, que optan por satisfacer sus necesidades de ocio.

\section{La gestión de la animación hotelera}

Cualquier enfoque de gestión, incorpora, de una u otra forma, tanto, las funciones generales que la sustenta: planificación, organización, ejecución, regulación y control, como el enfoque de servicio, que condiciona dicha actividad.

En este sentido, a la autora de este trabajo, le resulta más conveniente afiliarse a la definición de servicio ofrecida por la Ulacia (2015), donde lo interpreta como la integración de tangibles e intangibles dentro de actividades organizadas para producir una experiencia positiva en el cliente, por lo que la creación de cualquier producto-servicio, en este caso, de animación, debe partir de una orientación que refleje los beneficios que se desean brindar al segmento de mercado, al que se destina dicho producto. Esta orientación es conocida por los teóricos del tema servicios como Concepto de Servicio o más simplemente llamado en la práctica, Concepto.

Según Fisher (1994), el Concepto es “...la concepción teórica previa al servicio que se desea brindar, expresado mediante la precisión cualitativa y cuantitativa de los componentes del servicio...", Vale recordar que, los componentes del servicio a los que se hace referencia son los elementos tangibles e intangibles que componen el mismo. En relación a las funciones generales, la de planificación y de organización, en el tratamiento de la animación hotelera, se elabora un 
documento rector para su desarrollo denominado, Proyecto Operativo de Animación (POA) ${ }^{4}$, en el cual se establece la interrelación entre los recursos de la entidad y los de su entorno, para su explotación en función de la animación, se fundamenta atendiendo al Concepto previamente establecido, el cual rige estratégicamente las operaciones del establecimiento.

Para la elaboración del POA, después de haber analizado las propuestas metodológicas de algunos autores, esta autora considera que deben seguirse los 9 pasos que a continuación aparecen.

1. Datos generales de la instalación turística (Nombre del establecimiento ${ }^{5}$, localización, características generales (conceptualización, estándares...)

2. Análisis interno de la animación. El mismo comprende:

- Valoración de los recursos del establecimiento y del entorno

- Identificación de potencialidades que tiene la entidad para la animación

- Identificación de las potencialidades de los recursos humanos para apoyar activamente, las actividades de animación

- Selección de las potencialidades de los recursos del patrimonio con el interés de integrarlas en la animación

- Explotación, en función de la animación, de los recursos que identifican al establecimiento

3. Definición de los objetivos para el desarrollo de la animación.

4. Definición de la clientela y sus segmentos por edades (análisis ociotípicas).

5. Estructuración de las actividades de animación por programas basados en los segmentos de la clientela.

6. Definición de los programas de animación que produzcan mejor imagen para los establecimientos, según sus estándares y atributos.

7. Evaluación de las necesidades materiales, humanas y de equipamiento respecto a los programas de actividades de animación.

8. Análisis pre factibilidad del proyecto.

9. Aprobación del proyecto por el director.

10. Evaluación y control.

Como parte de esta función (Planificación), y derivado del POA, está también la elaboración de los Programas de Animación, uno de los documentos rectores de esta actividad (punto 6), posteriormente de su puesta en valor, se aplicarán herramientas de retroalimentación que permitirán evaluar la efectividad de los mismos dentro del proyecto.

La tabla que aparece a continuación, se refiere a los criterios emitidos por dos especialistas en la animación de Cuba, donde se muestra la diferencia que existe entre el programa de animación y el POA. Tabla No 1

${ }^{4}$ Aparece en Ulacia Oviedo, Zoila. Dossier I y II. Selección de lecturas sobre temas de Animación Turística, Centro de Estudios Turísticos. Universidad de la Habana, 2006, en la página 21 artículo "El proyecto operativo de animación en los hoteles" por el MSc. Enrique de la Tejera Segura.

${ }^{5}$ La palabra establecimiento es usada por la autora para hacer referencia a la entidad turística de que se trate a la hora de elaborar un POA, dígase hotel, y/o diferentes entidades de ocio del sistema turístico cubano. 
Tabla 1. Comparación entre programa de animación y el POA

\section{Programa de Animación}

Proyecto Operativo de Animación (POA)

Según Blanco Farías (2008) plantea que la

Según De la Ribera (1992) los programas de elaboración de un proyecto operativo de animación turística constituyen los documentos, que reflejan de forma ordenada, organizada y equilibrada las acciones diarias a desarrollar durante un período de tiempo en una instalación, con el propósito de cumplir las expectativas de los clientes para quienes va dirigida. animación, garantiza el desarrollo de los productos de animación y de los recursos humanos en conjunto. Este proyecto establece los objetivos que se deben perseguir, mediante medios de comunicación eficientes, que conduzcan hacia el rescate de los valores humanos y los recursos del patrimonio cultural y turístico.

Fuente: Definiciones tomadas de Luis Esteban de la Ribera Blanco y Margarita Blanco Faria

Según lo expuesto, la autora concluye que el POA es un sistema que permite interactuar los recursos del entorno con los del establecimiento, en función a la creación y el desarrollo de productos-animación, expresados en programas de actividades organizadas, que vienen siendo la razón de ser del mismo, con objetivos encaminados al logro de la satisfacción de las necesidades de ocio del cliente demandante.

Los programas de animación como expresión determinante de un POA, tienen que tener ciertas cualidades que responden al entorno natural y social en el cual se van a desarrollar, al tipo de segmentos demandantes según sus gustos y preferencias, el tiempo de estancia de estos en los establecimientos de alojamiento.

Las actividades deben abarcar: mañana, tarde y noche, teniendo en cuenta el espacio a realizarse, teniendo en cuenta el balance de los clímax dados por la variedad de ofertas recreativas y por la intensidad de las mismas, garantizando la eficacia, además, tener disponibilidad de recursos materiales y humanos, así como, contar con un presupuesto que permita ejecutar dicho programa con calidad.

Así mismo, se elaboran programas generales, programas temáticos y programas emergentes, estos últimos se diseñan para situaciones específicas, la ocurrencia de ciclones, lluvias intensas, falta de fluido eléctrico, incluso problemas de transportación, entre otras razones que por tales imprevistos no se puedan ejecutar las actividades programadas.

Las actividades de animación del programa, están respaldadas por la Ficha Técnica y el Guion, los cuales constituyen documentos rectores de dicha actividad. Es importante destacar que todo lo anterior debe estar en correspondencia al manual de operaciones de la cadena y los estándares que cumplen las marcas hoteleras. 


\section{La marca y su asociación con la animación hotelera. El rediseño de marcas}

En contextos tan competitivos y cambiantes como es la industria del turismo, la marca tiene un alto valor estratégico para toda empresa que desee desempeñarse con éxito, siendo uno de los tantos activos que conforman el conjunto de intangibles del marketing.

Según Domínguez (2005), viéndolo de forma simple y precisa, la marca "es la manera en que los clientes relacionan en su mente productos y servicios que compiten entre si'”. Es decir, la marca, brinda una realidad material, que tiene que ver con el nombre, y el imagotipo (logotipo), a través del cual el emisor trata de identificar y diferenciar sus productos para su desarrollo en el mercado a través de sus atributos, asociados a la imagen y al conjunto de signos emitidos por ella a través del producto.

Esto tiene que ver, indiscutiblemente, con aspectos comunicativos, extra verbales, para que el consumidor pueda identificar y diferenciar un producto de otro y se motive a realizar la compra de uno u otro, pero el tema del imagotipo no es precisamente la cuestión a tratar en esta investigación. Como refieren muchos autores la marca es una promesa y no basta que la propuesta satisfaga necesidades y expectativas de los clientes, sino que, tiene que despertar motivación y además crear lealtad estable y duradera.

Philip Kotler (2011) apunta que, "construir una marca no es sólo darle un nombre a un producto, es generar una experiencia de forma consistente a los compradores, a través de un conjunto especifico de características, beneficios y servicios".

Ahora bien, ¿qué pasa cuando la marca no transmite los atributos, no solo mediante su imagotipo, sino también mediante los servicios que brinda el producto? En estos casos, cuando no se cumple el papel que debe jugar la marca, es preciso un rediseño de la misma, entendiéndose por rediseño, la recreación de algo, remodelación, la modificación parcial y gradual, en este caso, del producto a través de los atributos.

Desde el punto de vista de la gestión empresarial significa, hacer cambios de forma gradual del fenómeno en cuestión, sin embargo, no es así con la reingeniería, que implica una modificación total y drástica de dicho producto, lo que comprende, la aparición de nuevos atributos, incluso otro imagotipo.

De manera tal que se parte del exhaustivo análisis de los atributos existentes con vista a identificar aquellos que no están funcionando en el mercado, significa entonces, modificar, innovar algún proceso, realizar una mejora.

El hecho de que el Concepto de la marca deba tributar a la animación se reafirma con la idea de Kotler (2011) cuando plantea "que las marcas propias tienen a su favor la posibilidad de 
innovar...", cabe preguntarse cuál es la diferencia que existe entre la marca objeto de estudio y la experiencia que evoca a través de la animación, que hace que el cliente supere las expectativas, piense que va a obtener valor si opta por ella y no por otra.

La animación en los hoteles bajo la marca Club Amigo, puede describirse tomando en consideración seis criterios fundamentales, que permiten asumir una idea más clara de este fenómeno, tales son:

- La conceptualización de la marca

- Manual operativo de animación turística y desarrollo sustentable

- Programas y actividades de animación

- El personal vinculado a la actividad

- Los recursos materiales y el presupuesto para el desarrollo de la actividad en su conjunto

- Los niveles de satisfacción del cliente hacia la animación

En este caso solo se toma en consideración la comparación que se realiza con la marca Brisas, partiendo de la conceptualización de ambas, lo cual se muestra en el siguiente cuadro comparativo.

Tabla 4. Cuadro comparativo de la conceptualización de las marcas Brisas y Club Amigo

\section{Conceptualización de la marca Club Amigo \\ Conceptualización de la marca Brisas}

En la conformación de su diseño e imagen, deben prevalecer elementos de cubanía, dados por la presencia de objetos utilitarios y decorativos representativos de la cultura nacional, que a su vez destaquen los valores y manifestaciones artísticas de la localidad donde se encuentre ubicada cada instalación. Todo ello, integrado armónicamente al contexto caribeño, en consonancia con el slogan que identifica esta marca: El más amistoso del Caribe, para lo cual se emplearán colores vivos y contrastantes.

Unido a lo anterior, la animación y la recreación constituyen factores determinantes de este producto turístico, cuya realización no se limita al equipo de animación, sino cuenta además con la activa participación de la mayor parte de los trabajadores del hotel.
En la conformación de su diseño e imagen, deben prevalecer elementos de cubanía, dados por la presencia de objetos utilitarios y decorativos representativos de la cultura nacional, que a su vez destaquen los valores y manifestaciones artísticas de la localidad donde se encuentre ubicada cada instalación. Todo ello, integrado armónicamente al contexto caribeño, en consonancia con el slogan que identifica esta marca: Fantasía incluida con sabor cubano, para lo cual se emplearán colores vivos y contrastantes.

Variadas y sistemáticas opciones de animación, recreación $y$ actividades deportivas. Representan componentes determinantes del producto, cuya realización no se limita al equipo de animación, sino que están respaldadas con la activa participación de la mayor parte de los trabajadores de cada instalación.

Fuente: Información extraída de los manuales operativos de las marcas Brisas y Club Amigo 
El contenido que se muestra en la tabla conduce a concluir que la conceptualización de la marca es reiterativa en su significado en una y otra y, por tanto, la animación, presenta insuficiencias y limitaciones que le afectan negativamente en un buen posicionamiento de esta en el mercado.

Por todo lo anterior se arriba al criterio que la problemática fundamental de la marca objeto de estudio, está determinada en que, no se percibe la correspondencia entre los atributos de la misma y el producto, lo que trae por consecuencia que se brinda información distorsionada al cliente.

\section{Conclusiones}

- El análisis de las distintas definiciones sobre el fenómeno de la animación turística permitió esclarecer criterios y emitir juicios oportunos para la problemática que se evidencia sobre la no diferenciación de marcas.

- Los usos de los métodos teóricos de investigación permitieron esclarecer que la conceptualización de la marca objeto de estudio es reiterativa en su significado, por lo que se toma en consideración el rediseño de la misma con vista a solucionar un problema.

\section{Referencias Bibliográficas}

Abreu Hernández, Pedro Pablo. (2007) en el artículo Animación y Recreación Turística. No es lo mismo un animador turístico que un guía turístico. (Fecha de acceso: sept/11 de 2015)

Blanco Faria, Margarita P. (diciembre 2008). Dossier Gestión de la Animación y Recreación. Universidad de Matanzas. Cuba.

De la Ribera Blanco, Luis. Material de Apoyo: Animación y Recreación Turística. Dirección Nacional de Capacitación INTUR, 1992.

Fisher, R. (1994). El Desafío del Servicio. Primera edición, Material del Seminario-Taller del mismo nombre, Chile.

Kotler, Philip (2011). Marketing para el Turismo. Edición Pearson. Madrid.

Manuales operativos de recreación y animación de las marcas Brisas y Club Amigo. (2016). Grupo Hotelero Cubanacán. S.A. Cuba.

Parreño, J.; Ruiz, E. y Casado, A. (2008). Dirección comercial: los instrumentos del marketing. 1ra Edición. Edit. Club Universitario. España. 
Pastor Raola, Roberto (2017). Informe sobre los resultados del estudio de efectividad de la animación, dictado en el Taller Nacional de Calidad del MINTUR. La Habana.

Puertas, Xavier (2010). Animación en el ámbito turístico. Ciclo Formativos FP Grado Superior de Hostelería y Turismo. Editorial Síntesis S.A. Madrid. España. p. 29-78

Saavedra Chávez. A (2010). Plan de capacitación para el desarrollo profesional de los recursos humanos que se desempeñan como animadores de las actividades recreativas físicas en los hoteles Cubanacán S.A. del Destino Jardines de Rey. Tesis de Maestría. Cuba.

Ulacia Oviedo, Z. (2015). Sistemas de Servicio en la Hospitalidad. Texto docente. Facultad de Turismo. Edit. Félix Varela, Universidad de La Habana, Cuba.

José Ignacio Domínguez (2005). Posicionamientos de marcas (marzo/ 2016). Recuperado: http://www.gestiopolis.com/Artículo. 


\section{Para citar el artículo indexado.}

Sánchez Romero, J. (2019). Animación hotelera, una perspectiva de diferenciación de marcas. Bases $\begin{array}{lllll}\text { conceptuales y metodológicas. } & \text { Explorador } & \text { Digital, } & \text { 3(4), }\end{array}$ https://doi.org/10.33262/exploradordigital.v3i4.917

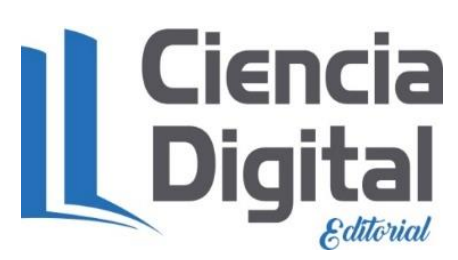

El artículo que se publica es de exclusiva responsabilidad de los autores y no necesariamente reflejan el pensamiento de la Revista Explorador Digital.

El articulo queda en propiedad de la revista y, por tanto, su publicación parcial y/o total en otro medio tiene que ser autorizado por el director o editor de la Revista Explorador Digital.

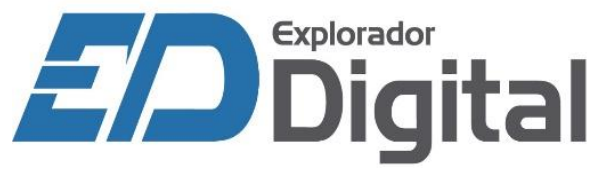

